Pacific Journal of Mathematic 


\title{
DISTRIBUTING TENSOR PRODUCT OVER DIRECT PRODUCT
}

\author{
K. R. GOODEARL
}

This paper is an investigation of conditions on a module $A$ under which the natural map

$$
A \otimes\left(\Pi C_{\alpha}\right) \longrightarrow \Pi\left(A \otimes C_{\alpha}\right)
$$

is an injection. The investigation leads to a theorem that a commutative von Neumann regular ring is self-injective if and only if the natural map

$$
\left(\Pi F_{\alpha}\right) \otimes\left(\Pi G_{\beta}\right) \longrightarrow \Pi\left(F_{\alpha} \otimes G_{\beta}\right)
$$

is an injection for all collections $\left\{F_{\alpha}\right\}$ and $\left\{G_{\beta}\right\}$ of free modules. An example is constructed of a commutative ring $R$ for which the natural map

$$
R[[s]] \otimes R[[t]] \longrightarrow R[[s, t]]
$$

is not an injection.

$R$ denotes a ring with unit, and all $R$-modules are unital. All tensor products are taken over $R$.

We state for reference the following theorem of H. Lenzing [2, Satz 1 and Satz 2]:

TheOREM L. (a) $A$ right $R$-module $A$ is finitely generated if and only if for any collection $\left\{C_{\alpha}\right\}$ of left $R$-modules, the natural map $A \otimes \Pi C_{\alpha} \rightarrow \Pi\left(A \otimes C_{\alpha}\right)$ is surjective.

(b) A right $R$-module $A$ is finitely presented if and only if for any collection $\left\{C_{\alpha}\right\}$ of left $R$-modules, the natural map $A \otimes \Pi C_{\alpha} \rightarrow$ $\Pi\left(A \otimes C_{\alpha}\right)$ is an isomorphism.

THEOREM 1. For any right $R$-module $A$, the following conditions are equivalent:

(a) If $\left\{C_{\alpha}\right\}$ is any collection of flat left $R$-modules, then the natural map $A \otimes \Pi C_{\alpha} \rightarrow \Pi\left(A \otimes C_{\alpha}\right)$ is an injection.

(b) There is a set $X$ of cardinality at least card $(R)$ such that the natural map $A \otimes R^{X} \rightarrow A^{X}$ is an injection.

(c) If $B$ is any finitely generated submodule of $A$, then the inclusion $B \rightarrow A$ factors through a finitely presented module.

Note that condition (c) always holds when $R$ is right noetherian, for then all finitely generated submodules of $A$ are finitely presented.

Proof. (b) $\Rightarrow$ (c): If $R$ is finite, then it is right noetherian and 
(c) holds. Thus we may assume that $R$ is infinite.

Let $f: F \rightarrow A$ be an epimorphism with $F_{R}$ free, and set $K=\operatorname{ker} f$. There is a finitely generated submodule $G$ of $F$ such that $f G=B$.

We have a commutative diagram with exact rows as follows (Diagram I):

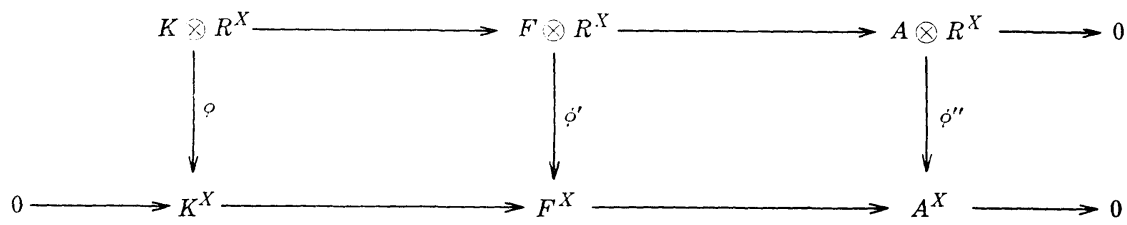

\section{DIAGRAM I}

Since $G$ is finitely generated, $G^{x} \leqq \phi^{\prime}\left(F \otimes R^{X}\right)$. A short diagram chase (using the injectivity of $\left.\phi^{\prime \prime}\right)$ shows that $(G \cap K)^{X} \leqq \phi\left(K \otimes R^{X}\right)$. card $(G) \leqq \operatorname{card}(R)$ because $R$ is infinite, hence card $(G \cap K) \leqq$ card $(X)$. Thus there is a surjection $\alpha \mapsto g_{\alpha}$ of $X$ onto $G \cap K$. The element $g=\left\{g_{\alpha}\right\}$ in $(G \cap K)^{X}$ must be the image under $\phi$ of some element $h_{1} \otimes r_{1}+\cdots+h_{n} \otimes r_{n}$ in $K \otimes R^{X}$. It follows easily that $G \cap K$ is contained in the submodule $H$ of $K$ generated by $h_{1}, \cdots, h_{n}$. Note that $G \cap H=G \cap K$.

$G+H$ is contained in some finitely generated free submodule $F_{0}$ of $F$. The map $f$ induces a monomorphism of $G /(G \cap H)$ into $A$, and this monomorphism factors through the finitely presented module $F_{0} / H$. Since $f G=B$, the inclusion $B \rightarrow A$ also factors through $F_{0} / H$.

(c) $\Rightarrow$ (a): Consider any $x$ belonging to the kernel of the natural map $\phi: A \otimes \Pi C_{\alpha} \rightarrow \Pi\left(A \otimes C_{\alpha}\right)$. There is a finitely generated submodule

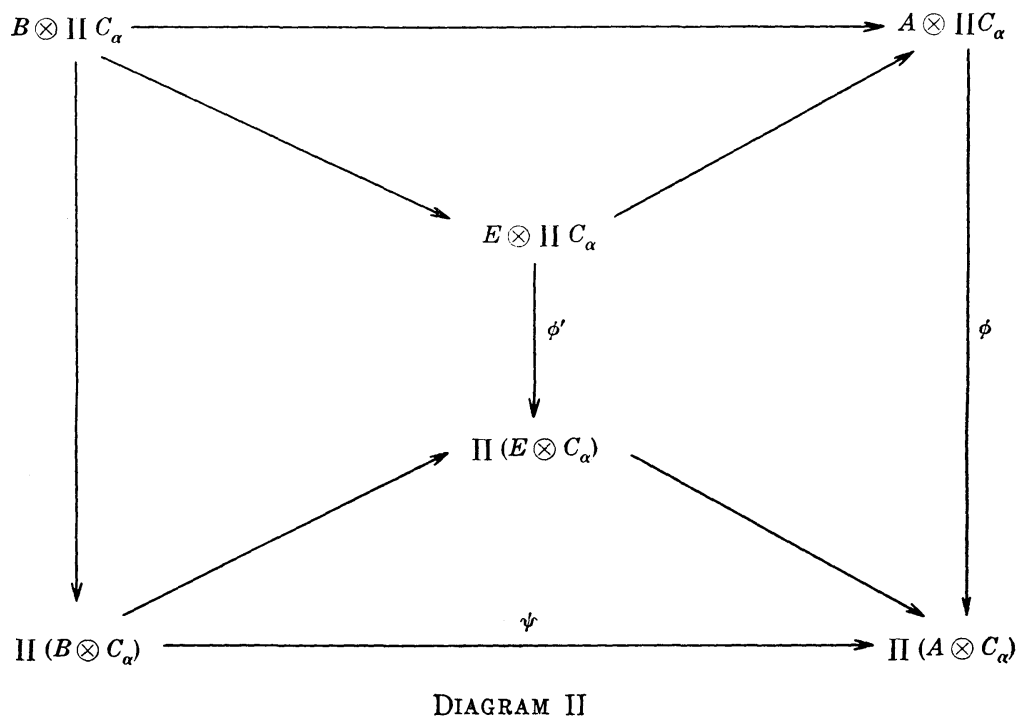


$B$ of $A$ such that $x$ is in the image of the map $B \otimes \Pi C_{\alpha} \rightarrow A \otimes \Pi C_{\alpha}$. By (c), the inclusion $B \rightarrow A$ factors through some finitely presented module $E$.

We have a commutative diagram as follows (Diagram II):

$\phi^{\prime}$ is an isomorphism by Theorem $\mathrm{L}$, and $\psi$ is a monomorphism because all the $C_{\alpha}$ 's are flat. Another diagram chase now shows that $x=0$.

CoRollary. Suppose that $R$ is (von Neumann) regular. For any right $R$-module $A$, the following conditions are equivalent:

(a) If $\left\{C_{\alpha}\right\}$ is any collection of left $R$-modules, then the natural map $A \otimes \Pi C_{\alpha} \rightarrow \Pi\left(A \otimes C_{\alpha}\right)$ is an injection.

(b) There is a set $X$ of cardinality at least card $(R)$ such that the natural map $A \otimes R^{X} \rightarrow A^{X}$ is injective.

(c) All finitely generated submodules of $A$ are projective.

Proof. (b) $\Rightarrow$ (c): If $B$ is a finitely generated submodule of $A$, then Theorem 1 says that the inclusion $B \rightarrow A$ factors through a finitely presented module $E$. $E$ is flat (because $R$ is regular) and hence is projective. Thus $B$ can be embedded in a projective module. Since $R$ is semihereditary, $B$ must be projective.

(c) $\Rightarrow$ (a): All the $C_{\alpha}$ 's are flat (since $R$ is regular), and all finitely generated submodules of $A$ are finitely presented, so this follows directly from Theorem 1.

THEOREM 2. Assume that $R$ is a commutative regular ring. Then the following conditions are equivalent:

(a) If $\left\{F_{\alpha}\right\}$ and $\left\{G_{\beta}\right\}$ are any collections of free $R$-modules, then the natural map $\left(\Pi F_{\alpha}\right) \otimes\left(\Pi G_{\beta}\right) \rightarrow \Pi\left(F_{\alpha} \otimes G_{\beta}\right)$ is an injection.

(b) There is a set $X$ of cardinality at least card $(R)$ such that the natural map $R^{X} \otimes R^{X} \rightarrow R^{X \times X}$ is an injection.

(c) $R$ is injective as a module over itself.

Proof. $\quad(b) \Rightarrow$ (c): By [1, Theorem 2.1], it suffices to show that any finitely generated nonsingular $R$-module $B$ is projective.

[1, Lemma 2.2] says that we can embed $B$ in a finite direct sum $Q_{1} \oplus \cdots \oplus Q_{n}$, where each $Q_{i}$ is a copy of the maximal quotient ring $Q$ of $R$. Then $B$ can be embedded in a direct sum $B_{1} \oplus \cdots \oplus B_{n}$, where $B_{i}$ is a finitely generated $R$-submodule of $Q_{i}$. Since $R$ is semihereditary, $B$ will be projective provided each $B_{i}$ is projective. Thus without loss of generality we may assume that $B$ is an $R$ submodule of $Q$.

Let $b_{1}, \cdots, b_{n}$ generate $B$. Since $R$ is an essential submodule of $Q$, there is an essential ideal $I$ of $R$ such that $b_{i} I \leqq R$ for all $i$. 
Since $R$ is commutative, the multiplications by the elements of $I$ induce homomorphisms of $B$ into $R$. Together, these homomorphisms induce a homomorphism $f: B \rightarrow R^{I} . \quad Q$ is a nonsingular $R$-module because it has the nonsingular $R$-module $R$ as an essential submodule. Thus no nonzero element of $B$ is annihilated by $I$; i.e., $f: B \rightarrow R^{I}$ is an injection. Since $\operatorname{card}(I) \leqq \operatorname{card}(R) \leqq \operatorname{card}(X)$, there must also be an embedding of $B$ into $R^{x}$.

Since the natural map $R^{X} \otimes R^{X} \rightarrow\left(R^{Y}\right)^{X}$ is injective by (b), the corollary to Theorem 1 says that all finitely generated submodules of $R^{X}$ are projective. Thus $B$ must be projective.

(c) $\Rightarrow$ (a): By [1, Theorem 2.1], all finitely generated nonsingular $R$-modules are projective. Since $R_{R}$ is nonsingular, $\Pi F_{\alpha}$ is nonsingular; thus all finitely generated submodules of $\Pi F_{\alpha}$ are projective. By the corollary to Theorem 1 , the natural map $\left(\Pi F_{\alpha}\right) \otimes\left(\Pi G_{\beta}\right) \rightarrow$ $\Pi_{\beta}\left[\left(\Pi F_{\alpha}\right) \otimes G_{\beta}\right]$ is an injection. Likewise, each of the maps $\left(\Pi F_{\alpha}\right) \otimes G_{\beta} \rightarrow$ $\Pi_{\alpha}\left(F_{\alpha} \otimes G_{\beta}\right)$ is injective. Thus the map $\left(\Pi F_{\alpha}\right) \otimes\left(\Pi G_{\beta}\right) \rightarrow \Pi\left(F_{\alpha} \otimes G_{\beta}\right)$ must be injective.

In particular, Theorem 2 asserts that if $R$ is a countable commutative regular ring which is not self-injective, then the natural map $R^{X} \otimes R^{X} \rightarrow R^{X \times X}$ is not an injection for any infinite set $X$. For example, let $F_{1}, F_{2}, \cdots$ be a countable sequence of copies of some countable field $F$; let $R$ be the subalgebra of $\Pi F_{n}$ generated by 1 and $\oplus F_{n}$. $R$ is obviously a countable commutative regular ring. Since $\Pi F_{n}$ is a proper essential extension of $R_{R}, R_{R}$ is not injective.

If $N$ is the set of natural numbers, then the natural map $R^{N} \otimes R^{N} \rightarrow R^{N \times N}$ is not an injection. Thus the tensor product of two one-variable power series rings, $R[[s]] \otimes R[[t]]$, is not embedded in $R[[s, t]]$ by the natural map.

\section{REFERENCES}

1. V. C. Cateforis, On regular self-injective rings, Pacific J. Math., 30 (1969), 39-45.

2. H. Lenzing, Endlich präsentierbare Moduln, Arch. der Math., 20 (1969), 262-266.

Received July 28, 1971.

UNIVERSITY OF WASHINGTON

Author's current address: UNIVERSITY OF UTAH 


\section{PACIFIC JOURNAL OF MATHEMATICS}

\section{EDITORS}

\section{H. SAMElson}

Stanford University

Stanford, California 94305

C. R. Новву

University of Washington

Seattle, Washington 98105
J. DugunduI

Department of Mathematics University of Southern California

Los Angeles, California 90007

RICHARD ARENS

University of California

Los Angeles, California 90024

\section{ASSOCIATE EDITORS}
E. F. BECKENBACH
B. H. NeUmanN
F. WOLF
K. YosHIDA

\section{SUPPORTING INSTITUTIONS}

UNIVERSITY OF BRITISH COLUMBIA

UNIVERSITY OF SOUTHERN CALIFORNIA

CALIFORNIA INSTITUTE OF TECHNOLOGY

STANFORD UNIVERSITY

UNIVERSITY OF CALIFORNIA

UNIVERSITY OF TOKYO

MONTANA STATE UNIVERSITY

UNIVERSITY OF UTAH

UNIVERSITY OF NEVADA

WASHINGTON STATE UNIVERSITY

NEW MEXICO STATE UNIVERSITY

OREGON STATE UNIVERSITY

UNIVERSITY OF OREGON

OSAKA UNIVERSITY

UNIVERSITY OF WASHINGTON

* *

AMERICAN MATHEMATICAL SOCIETY

NAVAL WEAPONS CENTER 


\section{Pacific Journal of Mathematics}

\section{Vol. 43, No. $1 \quad$ March, 1972}

Alexander (Smbat) Abian, The use of mitotic ordinals in cardinal

arithmetic ....................................... 1

Helen Elizabeth. Adams, Filtrations and valuations on rings ......... 7

Benno Artmann, Geometric aspects of primary lattices .............. 15

Marilyn Breen, Determining a polytope by Radon partitions ........... 27

David S. Browder, Derived algebras in $L_{1}$ of a compact group .......... 39

Aiden A. Bruen, Unimbeddable nets of small deficiency .............. 51

Michael Howard Clapp and Raymond Frank Dickman, Unicoherent

compactifications ............................... 55

Heron S. Collins and Robert A. Fontenot, Approximate identities and the strict topology ................................... 63

R. J. Gazik, Convergence in spaces of subsets................. 81

Joan Geramita, Automorphisms on cylindrical semigroups ........... 93

Kenneth R. Goodearl, Distributing tensor product over direct product ..... 107

Julien O. Hennefeld, The non-conjugacy of certain algebras of

operators ................................... 111

C. Ward Henson, The nonstandard hulls of a uniform space ........... 115

M. Jeanette Huebener, Complementation in the lattice of regular

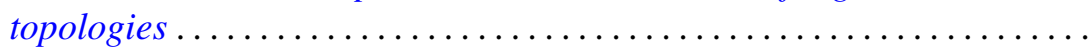

Dennis Lee Johnson, The diophantine problem $Y^{2}-X^{3}=A$ in a

polynomial ring .................................... 151

Albert Joseph Karam, Strong Lie ideals . . . . . . . . . . . . . . . . . . . . 157

Soon-Kyu Kim, On low dimensional minimal sets ............... 171

Thomas Latimer Kriete, III and Marvin Rosenblum, A Phragmén-Lindelöf

theorem with applications to $M(u, v)$ functions ..... . .

William A. Lampe, Notes on related structures of a universal algebra . . . . 189

Theodore Windle Palmer, The reducing ideal is a radical .

207

Kulumani M. Rangaswamy and N. Vanaja, Quasi projectives in abelian and module categories ................................ 221

Ghulam M. Shah, On the univalence of some analytic functions ......... 239

Joseph Earl Valentine and Stanley G. Wayment, Criteria for Banach

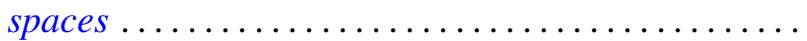

Jerry Eugene Vaughan, Linearly stratifiable spaces ............... 253

Zbigniew Zielezny, On spaces of distributions strongly regular with respect to partial differential operators ..................... 